\title{
Fauna de Alticini (Newman) (Coleoptera, Chrysomelidae, Galerucinae) em diferentes estágios sucessionais na Floresta com Araucária do Paraná, Brasil: diversidade e estimativa de riqueza de espécies ${ }^{1}$
}

\author{
Adelita Maria Linzmeier ${ }^{2}$, Cibele Stramare Ribeiro-Costa² \& Renato Contin Marinoni² \\ ${ }^{1}$ Contribuição número 1617 do Departamento de Zoologia da Universidade Federal do Paraná. \\ ${ }^{2}$ Departamento de Zoologia, Universidade Federal do Paraná, Caixa Postal 19020, 81531-980 Curitiba-PR, Brasil. Bolsistas do CNPq. \\ alinzmeier@yahoo.com.br,stra@ufpr.br,rcmari@ufpr.br
}

\begin{abstract}
Alticini (Newman) (Coleoptera, Chrysomelidae, Galerucinae) fauna in different successional stages in an Araucaria Forest of Paraná, Brazil: diversity and estimation of species richness. Members of the Alticini were collected at five locations: an edge, an area of unmanaged Araucaria reforestation, and in three successional stages of Araucaria Forest in the state of Paraná. Collecting, using malaise traps, was carried out weekly from September 1999 to August 2001, in Vila Velha State Park, near the city of Ponta Grossa, Paraná. Collections included 1,891 specimens of 106 species in the Alticini. The highest richness and the lowest species abundance were in the forest margin. Richness was highest in young, and lowest in older successional stages. The richness estimators indicated a possible increase of seven to 50 Alticini species in Vila Velha. The proportional abundance of Chrysomelidae/Coleoptera decreases with increasing degree of succession, and therefore may serve as an indicator of environmental quality.
\end{abstract}

KEYWORDS. Abundance; bioindicator; malaise trap; survey.

\begin{abstract}
RESUMO. Fauna de Alticini (Newman) (Coleoptera, Chrysomelidae, Galerucinae) em diferentes estágios sucessionais na Floresta com Araucária do Paraná, Brasil: diversidade e estimativa de riqueza de espécies. A fauna de Alticini foi levantada em cinco áreas: uma de borda de mata, uma com povoamento de auracária sem manejo e três em diferentes estágios sucessionais, na Floresta com Araucária do Paraná. As coletas foram realizadas com armadilhas malaise, semanalmente, de setembro de 1999 a agosto de 2001, no Parque Estadual de Vila Velha, Ponta Grossa, PR. Foram coletados 1.891 Alticini em 106 espécies. A área de borda apresentou a maior riqueza e a menor abundância. Nas áreas em sucessão, a riqueza aumentou nos estágios iniciais e diminui no estágio mais avançado. As estimativas de riqueza apontam para um aumento de sete a 50 espécies de Alticini em Vila Velha. A proporção de Chrysomelidae/Coleoptera diminuiu com o aumento do grau de sucessão vegetal das áreas, podendo tal relação, servir como indicador de qualidade ambiental.
\end{abstract}

PALAVRAS-CHAVE. Abundância; bioindicador; levantamento; malaise.

O Parque Estadual de Vila Velha é uma unidade de conservação de proteção integral do Estado do Paraná onde predomina a Floresta Ombrófila Mista ou Floresta com Araucária. Nesse local, mais especificamente em uma área caracterizada como um capão de araucárias foram reconhecidas cinco áreas alteradas de diferentes maneiras por ação antrópica. Resultados de inventários conduzidos nessas áreas, com base na riqueza e abundância das famílias e morfo-espécies de Coleoptera, corroboraram os diferentes níveis de conservação ambiental das mesmas (Ganho \& Marinoni 2003; Marinoni \& Ganho 2003; Ganho \& Marinoni 2005) .

Em geral, espécies de diferentes níveis tróficos são afetadas diferentemente pela fragmentação dos habitats (Didham et al. 1998b). Neste sentido, Chrysomelidae e Alticini, como grupos essencialmente herbívoros e abundantemente coletados (Farrell \& Erwin 1988; Takizawa 1994; Wagner 1999; Novotný et al. 1999; Ganho \& Marinoni 2003), podem possuir importante significado para as avaliações das relações tróficas nos diferentes locais levantados e devem responder às alterações antrópicas. Estudos desenvolvidos como os de Morris (1980), Hutcheson (1990) e Marinoni \& Dutra (1997) verificaram que os Coleoptera herbívoros predominam em áreas mais degradadas, em início de regeneração, enquanto os grupos detritívoros e fungívoros predominam em áreas mais conservadas.
Assim, dada a grande riqueza de Alticini (Furth 1988; Scherer 1988), sua facilidade de coleta e possíveis respostas à degradação ambiental, este trabalho busca estabelecer relações entre este grupo e as condições florísticas de cada área em Vila Velha, sendo avaliados os seguintes itens: 1) riqueza e abundância; 2) proporção de Chrysomelidae/Coleoptera e Alticini/Coleoptera a fim de associar tais relações as diferentes condições florísticas das áreas; 3 ) estimativa da riqueza de Alticini com diferentes métodos; 4) presença de espécies comuns e raras nas áreas; 5) curva de acumulação de espécies.

\section{MATERIAL E MÉTODOS}

O levantamento foi realizado no Parque Estadual de Vila Velha, localizado no município de Ponta Grossa, Paraná, junto à Rodovia do Café, BR 376, $\mathrm{Km} 83$, a $880 \mathrm{~m}$ de altitude, durante o período de 30 de agosto de 1999 a 27 de agosto de 2001.

Foram selecionadas cinco áreas: 1) borda (Borda) entre a floresta e o campo, este mantido por manejo; 2) com plantio de araucária (Araucária), sem manejo de limpeza e corte; 3 ) em sucessão inicial a intemediária (Fase 1);4) em sucessão intermediária a avançada (Fase 2); 5) em sucessão avançada (Fase 3). No texto a Fase 1 é considerada como a menos conservada e a Fase 3 a mais conservada, estando a área Fase 2 em uma posição intermediária. 
Detalhes das áreas tais como, situação geográfica, características vegetais e distâncias entre os pontos de coleta, estão descritos em Ganho \& Marinoni (2003).

Em cada área foi instalada uma armadilha malaise (Townes 1972) sendo o material retirado semanalmente do frasco coletor.

Os Alticini foram identificados ao menor nível taxonômico possível, com base em bibliografia pertinente, por comparação com o material da Coleção de Entomologia Pe. Jesus Santiago Moure (DZUP), da Fundação Zoobotânica do Rio Grande do Sul (FZRS) e do Museu Anchieta, Rio Grande do Sul.

As informações referentes aos Alticini foram inseridas em um banco de dados gerenciado pelo programa MS Access 2000. O material está depositado na Coleção de Entomologia Pe. J. S. Moure do Departamento de Zoologia da Universidade Federal do Paraná.

Foi realizada a análise de variância ou o teste de KruskalWallis a 5\% de significância, com os dados de abundância de Alticini de cada uma das áreas para verificar se havia diferença nos valores entre os anos. A normalidade dos dados foi previamente verificada pelo teste de Kolmogorov-Smirnov (KS). Estas análises foram realizadas com o programa Statistica 6.0.

Para cada uma das áreas foram calculados os índices de diversidade de Brillouin (HB) e de Shannon (H'), dominância de Berger \& Parker (BP) e uniformidade de Berger \& Parker (UBP) (Magurran 2004). Para o cálculo do índice de Brillouin, os valores de $n$ fatorial foram obtidos pelo método proposto por Oliveira et al. (1998).

O termo singleton foi utilizado para as espécies que possuem um único exemplar e doubleton para as que possuem um único exemplar no conjunto das coletas realizadas (Colwell 2004).

Para se obter a estimativa da riqueza de espécies, foram utilizados os estimadores não-paramétricos, Chao 1, Chao 2 , Jack-Knife 1, Jack-Knife 2, Bootstrap e Michaelis-Menten, através do programa EstimateS: Richness Estimator, versão 7.00 (Colwell 2004). Foram utilizadas 50 casualizações com abundância de classes igual a 10. As estimativas foram realizadas apenas com os dados do primeiro ano de coleta (setembro/1999 a agosto/2000), num total de 52 semanas, para cada uma das áreas e para Vila Velha (somando-se todas as áreas). Da mesma forma, foram realizadas estimativas apenas com os dados do segundo ano de coleta (setembro/2000 a agosto/2001) e, do período como um todo, de setembro/1999 a agosto/2001, num total de 104 semanas de coleta.

\section{RESULTADOS E DISCUSSÃO}

Abundância. De setembro de 1999 a agosto de 2001, foram coletados 19.914 coleópteros, sendo 10.822 no primeiro ano (1999/2000) e 9.092 no segundo (2000/2001). A área Fase 2, em estágio intermediário a avançado de sucessão, foi a que apresentou maior número de coleópteros, seguida pelas áreas Borda, Araucária, Fase 3 e Fase 1 (Tabela I). Houve uma grande diferença no número de coleópteros de um ano para outro, principalmente nas áreas Fase 1 e Borda. Na área Fase1, houve uma diminuição de $68,83 \%$ na captura de Coleoptera porém não significativa segundo o teste de Kruskal-Wallis, e na área de Borda, houve um acréscimo de 44,32\%, também não significativo.

Como visto para Coleoptera, a menor abundância na área Fase1 no segundo ano também foi observada em outros grupos, como Lepidoptera (observação pessoal) e Diptera (L. Marinoni, comunicação pessoal), porém ainda há necessidade de maiores estudos para elucidar a causa de tal decréscimo.

Do total de Coleoptera coletado nos dois anos, 15,96\% pertence à Chrysomelidae (3.179 exemplares), família entre as mais abundantemente coletadas em diversos trabalhos em várias regiões e com diferentes métodos de coleta (Marinoni \& Dutra 1997; Wagner 1999; Barbosa et al. 2002; Ganho \& Marinoni 2003). Para Chrysomelidae, assim como para Coleoptera, a área Fase 2 foi a que apresentou a maior captura e a Fase 3, em estágio avançado de sucessão, a menor (Tabela I).

Como observado para Coleoptera, Chrysomelidae também teve uma variação no número de exemplares capturados de um ano para outro. Na Fase1, houve uma diferença significativa $\left(\mathrm{F}_{1,22}=6,98, \mathrm{p}<0,05\right)$ do primeiro para o segundo ano com diminuição de $70,37 \%$ dos crisomelídeos capturados. Ao contrário de Coleoptera, onde somente a área de Borda teve um acréscimo de um ano para outro, em Chrysomelidae tanto $\mathrm{a}$ área de Borda $(29,17 \%)$, como as áreas Fase $2(10,83 \%) \mathrm{e}$ Fase $3(13,26 \%)$ tiveram aumento de um ano para outro, porém os valores não foram estatisticamente significativos.

Durante os dois anos de amostragem foram coletados 1.891 exemplares de Alticini, 978 no primeiro ano e 913 no segundo. Considerando-se os dois anos em conjunto, observa-se que o maior número de indivíduos foi capturado na Fase 2 e o menor na área de Borda (Tabela I).

A abundância de Alticini nas áreas de Borda e Araucária se manteve praticamente constante de um ano para outro (Tabela I), porém na Fase 1, que se encontra em estágio inicial a intermediário de sucessão, houve um decréscimo significativo $\left(\mathrm{F}_{1,22}=13,25, \mathrm{p}<0,05\right)$ de $63,5 \%$. Já nas áreas Fase 2 e Fase 3, em estágios mais avançados de sucessão, houve um pequeno acréscimo não sendo estatisticamente significativo. Esse grupo foi o mais abundantemente coletado dentro de Chrysomelidae, perfazendo $59,5 \%$ do total amostrado.

Proporção de Chrysomelidae e Alticini. Verificando a proporção de Chrysomelidae/Coleoptera e de Alticini/ Coleoptera nas áreas em sucessão, observa-se que a proporção de Chrysomelidae diminui com o avanço do processo sucessional das áreas (Tabela II). Alticini não seguiu exatamente esse padrão, tendo um pequeno aumento da área Fase $1(12,43 \%)$ para a Fase $2(13,67 \%)$, porém, na área em estágio avançado ocorreu o menor valor dentre as três áreas em sucessão (Tabela II). Este aumento da proporção na área Fase 2 foi devido provavelmente a duas espécies muito abundantes nessa área, Phyllotrupes violaceomaculatus (Bechyné, 1958) e Syphraea olga Bechyné, 1955, já que Dinaltica gigia Bechyné, 1956, apesar de ter sido dominante 
Tabela I. Abundância (N) de Coleoptera, Chrysomelidae e Alticini e, riqueza (S) de Alticini capturados com malaise em cada um dos anos e durante todo o período de setembro/1999 a agosto/2001, em cinco áreas no Parque Estadual de Vila Velha, Ponta Grossa, Paraná.

\begin{tabular}{lccccc}
\hline & Áreas & Coleoptera & Chrysomelidae & Alticini & Alticini \\
& & $(\mathrm{N})$ & $(\mathrm{N})$ & $(\mathrm{N})$ & $(\mathrm{S})$ \\
\hline 9 & Borda & 1.699 & 216 & 94 & 39 \\
9 & Araucária & 1.817 & 267 & 140 & 16 \\
- & Fase 1 & 2.477 & 567 & 296 & 28 \\
0 & Fase 2 & 2.946 & 480 & 342 & 30 \\
0 & Fase 3 & 1.883 & 196 & 106 & 23 \\
\hline Total & & & & & \\
$1999-2000$ & & 10.822 & 1.726 & 978 & 84 \\
\hline 0 & Borda & 2.452 & 279 & 100 & 36 \\
0 & Araucária & 1.719 & 252 & 144 & 19 \\
- & Fase 1 & 772 & 168 & 108 & 10 \\
0 & Fase 2 & 2.612 & 532 & 418 & 28 \\
1 & Fase 3 & 1.537 & 222 & 143 & 22 \\
\hline Total & & & & & \\
$2000-2001$ & & 9.092 & 1.453 & 913 & 76 \\
\hline 9 & Borda & 4.151 & 495 & 194 & 55 \\
9 & Araucária & 3.536 & 519 & 284 & 26 \\
- & Fase 1 & 3.249 & 735 & 404 & 30 \\
0 & Fase 2 & 5.558 & 1.012 & 760 & 40 \\
1 & Fase 3 & 3.420 & 418 & 249 & 29 \\
\hline Total & & & & & \\
$1999-2001$ & & 19.914 & 3.179 & 1891 & 106 \\
\hline \multicolumn{7}{l}{} & & & &
\end{tabular}

nessa área, também ocorreu em grande número na área Fase 1 e, quando retirada da análise, a ordem dos valores permaneceu a mesma. Para a área de Araucária, os valores tanto para Chrysomelidae como para Alticini ficaram em uma posição intermediária entre as áreas Fase 2 e Fase 3. Já para a área de Borda, foram registradas as menores proporções tanto para Chrysomelidae como para Alticini (Tabela III).

Apesar de Alticini não ter mantido a diminuição gradual, da área em estágio inicial de sucessão para a área em estágio mais avançado, a partir dos valores percentuais de Chrysomelidae, que manteve tal relação, foi possível corroborar os trabalhos de Morris (1980), Hutcheson (1990), Marinoni \& Dutra (1997) e Ganho \& Marinoni (2005). Assim, onde predominam plantas herbáceas e arbustivas (estágios iniciais de sucessão) há dominância de coleópteros herbívoros e em ambientes em que espécies vegetais são de maior porte, propiciando um ambiente úmido, menos iluminado e com solo rico em materiais em decomposição (estágios avançados de sucessão), predominam coleópteros detritívoros e fungívoros como Staphylinidae, por exemplo.

Riqueza de espécies. Nas cinco áreas amostradas durante os dois anos foram capturadas 106 espécies de Alticini. Na área de Borda ocorreu a maior riqueza e na de Araucária, bastante sombreada pela copa das araucárias e que é a mais homogênea, a menor (Tabela III e IV). Ganho \& Marinoni (2005) também observaram a menor riqueza na área de Araucária para Chrysomelidae e na área Fase 3 para Coleoptera em geral, Cerambycidae e Curculionidae.
Somente a área de Araucária teve um pequeno incremento no número de espécies de Alticini no segundo ano de coleta. Ao contrário, todas as outras áreas apresentaram um menor número de espécies capturadas no segundo ano, principalmente a Fase1 onde foram capturadas somente 10 espécies (Tabela I).

Nas áreas em sucessão, a riqueza aumentou nos estágios iniciais, Fase 1 e Fase 2 e diminui no estágio mais avançado, Fase 3. Provavelmente nos estágios iniciais de sucessão há maior disponibilidade de recursos alimentares para os Alticini, permitindo que um maior número de espécies possa coexistir no local.

Dependendo do tipo do habitat adjacente, a borda pode exibir um aumento ou uma diminuição no número de espécies, comparado ao habitat original (Tscharntke \& Brandl 2004). No presente estudo a área de Borda apresentou a maior riqueza e a menor abundância (Tabela IV). Segundo Didham et al. (1998a), o grande número de espécies coletadas nas áreas de borda é o resultado do aumento da produtividade e do influxo de espécies das áreas vizinhas. Assim, a área de Borda amostrada deve possuir tanto espécies da área florestada como da área de campo e também espécies características do próprio local. Como esta área possui uma alta produtividade devido a alta insolação, isso deve contribuir para a formação de folhas jovens, de um grande número de espécies herbáceas encontradas na área de campo e na bordadura, as quais são a fonte principal de alimento para Alticini. A presença de um grande número de folhas em áreas florestadas não significa necessariamente abundância de alimento para os herbívoros, já que folhas maduras em comparação com folhas jovens contém mais toxinas e são menos nutritivas (Wolda 1978). Ganho \& Marinoni (2005), verificaram também que Chrysomelidae apresentou a maior riqueza na área de Borda. Já para Coleoptera, a maior riqueza foi na área Fase1. Cabe salientar que dentro de Coleoptera as espécies ocupam vários níveis tróficos, e cada grupo pode responder de forma diferente ao efeito de borda, enquanto que neste trabalho, somente os herbívoros (Alticini) foram avaliados.

Didham et al. (1998b), estudando os Coleoptera em um ambiente experimentalmente fragmentado na Amazônia Central, verificaram que a maioria das espécies encontradas mais próximas à borda foram raras ou moderadamente comuns, assim como no presente trabalho em que $41,8 \%$ das espécies (Tabela V) apresentaram somente um indivíduo. Espécies raras podem ser cosideradas do próprio local, ocasionais ou ainda podem

Tabela II. Porcentagem (\%) de Chrysomelidae e Alticini por Coleoptera, capturados de setembro/1999 a agosto/2001 com malaise, em cinco áreas no Parque Estadual de Vila Velha, Ponta Grossa, Paraná.

\begin{tabular}{lcc}
\hline Áreas & Chrysomelidae/Coleoptera & Alticini/Coleoptera \\
\hline Borda & 11,92 & 4,67 \\
Araucária & 14,68 & 8,03 \\
Fase 1 & 22,62 & 12,43 \\
Fase 2 & 18,21 & 13,67 \\
Fase 3 & 12,22 & 7,28 \\
\hline
\end{tabular}


Tabela III. Lista de espécies coletadas com malaise, em cinco áreas, no Parque Estadual de Vila Velha, Ponta Grossa, Paraná, de setembro/1999 a agosto/2001.

\begin{tabular}{|c|c|c|c|c|c|c|}
\hline Espécies & Borda & Araucária & Fase 1 & Fase 2 & Fase 3 & Total \\
\hline Acallepitrix coracina (Boheman, 1859) & 0 & 0 & 0 & 0 & 3 & 3 \\
\hline Acanthonycha chloroptera (Germar, 1824) & 5 & 2 & 6 & 3 & 17 & 33 \\
\hline Acanthonycha costatipennis Jacoby, 1905 & 9 & 3 & 3 & 13 & 26 & 54 \\
\hline Acanthonycha sp. & 1 & 7 & 0 & 6 & 0 & 14 \\
\hline Alagoasa coccineloides (Harold, 1847) & 0 & 0 & 1 & 0 & 0 & 1 \\
\hline Alagoasa libentina (Germar, 1824) & 0 & 2 & 0 & 0 & 0 & 2 \\
\hline Alagoasa parana Samuelson, 1985 & 3 & 0 & 0 & 0 & 0 & 3 \\
\hline Alagoasa plaumanni Bechyné, 1955 & 1 & 0 & 0 & 0 & 0 & 1 \\
\hline Alagoasa rotundicollis (Jacoby, 1905) & 6 & 0 & 3 & 0 & 0 & 9 \\
\hline Alagoasa scissa ab sordidula (Csiki, 1940) & 1 & 0 & 0 & 0 & 0 & 1 \\
\hline Alagoasa sp.1 & 1 & 2 & 0 & 0 & 0 & 3 \\
\hline Alagoasa sp.2 & 0 & 0 & 0 & 0 & 1 & 1 \\
\hline Alagoasa vittata (Harold, 1876) & 0 & 0 & 1 & 0 & 0 & 1 \\
\hline Altica bohumilae Bechyné, 1954 & 3 & 0 & 0 & 0 & 0 & 3 \\
\hline Alticini sp.04 & 8 & 12 & 0 & 1 & 2 & 23 \\
\hline Alticini sp. 21 & 1 & 0 & 0 & 0 & 0 & 1 \\
\hline Alticini sp. 23 & 1 & 0 & 0 & 0 & 0 & 1 \\
\hline Alticini sp.26 & 2 & 0 & 0 & 0 & 0 & 2 \\
\hline Alticini sp. 35 & 0 & 0 & 0 & 1 & 0 & 1 \\
\hline Alticini sp.41 & 0 & 0 & 0 & 35 & 1 & 36 \\
\hline Alticini sp. 45 & 0 & 0 & 3 & 6 & 1 & 10 \\
\hline Alticini sp.47 & 0 & 0 & 0 & 0 & 4 & 4 \\
\hline Alticini sp. 60 & 0 & 0 & 0 & 1 & 0 & 1 \\
\hline Alticini sp.67 & 0 & 0 & 8 & 0 & 0 & 8 \\
\hline Alticini sp.75 & 0 & 0 & 0 & 1 & 1 & 2 \\
\hline Alticini sp.90 & 0 & 0 & 0 & 1 & 0 & 1 \\
\hline Alticini sp.106 & 0 & 0 & 0 & 1 & 0 & 1 \\
\hline Alticini sp.110 & 1 & 0 & 0 & 0 & 0 & 1 \\
\hline Alticini sp. 125 & 0 & 0 & 0 & 1 & 0 & 1 \\
\hline Alticini sp.126 & 0 & 0 & 1 & 0 & 0 & 1 \\
\hline Alticini sp. 127 & 1 & 0 & 0 & 0 & 0 & 1 \\
\hline Asphaera auripennis Harold, 1876 & 1 & 0 & 0 & 0 & 0 & 1 \\
\hline Asphaera hilaris (Jacoby, 1905) & 3 & 3 & 0 & 0 & 0 & 6 \\
\hline Brasilaphtona dilutiventris umbraticeps Bechyné, 1956 & 0 & 0 & 2 & 0 & 0 & 2 \\
\hline Brasilaphtona octavia Bechyné, 1955 & 1 & 0 & 3 & 0 & 0 & 4 \\
\hline Brasilaphtona sp.1 & 0 & 3 & 1 & 0 & 3 & 7 \\
\hline Brasilaphtona sp.2 & 1 & 0 & 0 & 0 & 5 & 6 \\
\hline Capraita sp.1 & 0 & 0 & 0 & 12 & 0 & 12 \\
\hline Capraita sp. 2 & 0 & 0 & 0 & 1 & 2 & 3 \\
\hline Chaetocnema braziliensis Baly, 1877 & 0 & 0 & 0 & 0 & 1 & 1 \\
\hline Cornulactica jacobyi Bechyné, 1955 & 1 & 0 & 0 & 0 & 0 & 1 \\
\hline Coroicona sp. & 0 & 0 & 0 & 7 & 0 & 7 \\
\hline Dinaltica gigia Bechyné, 1956 & 2 & 2 & 187 & 269 & 3 & 463 \\
\hline Disonycha conjuncta (Germar, 1824) & 3 & 0 & 0 & 0 & 0 & 3 \\
\hline Dysonycha plaumanni C. Lima, 1954 & 1 & 0 & 0 & 0 & 0 & 1 \\
\hline Epitrix sp.1 & 1 & 0 & 0 & 0 & 1 & 2 \\
\hline Epitrix sp.2 & 1 & 0 & 0 & 0 & 0 & 1 \\
\hline Genaphtona yasmina Bechyné, 1955 & 0 & 0 & 0 & 0 & 7 & 7 \\
\hline Heikertingerella bimaculata (Baly, 1877) & 0 & 0 & 0 & 0 & 18 & 18 \\
\hline Heikertingerella ferruginea Duvivier, 1889 & 7 & 1 & 1 & 6 & 45 & 60 \\
\hline Heikertingerella sp.1 & 0 & 0 & 0 & 2 & 11 & 13 \\
\hline Heikertingerella sp. 2 & 0 & 0 & 0 & 0 & 3 & 3 \\
\hline Heikertingerella sp. 3 & 2 & 0 & 0 & 1 & 0 & 3 \\
\hline Heikertingerella sp.4 & 0 & 0 & 0 & 1 & 0 & 1 \\
\hline Heikertingerella sp.5 & 0 & 1 & 62 & 1 & 0 & 64 \\
\hline Heikertingerella sp.6 & 2 & 0 & 0 & 0 & 0 & 2 \\
\hline Heikertingerella sp.7 & 0 & 0 & 0 & 2 & 0 & 2 \\
\hline Heikertingerella sp. 8 & 0 & 0 & 28 & 0 & 0 & 28 \\
\hline Heikertingerella sp.9 & 0 & 0 & 0 & 0 & 3 & 3 \\
\hline
\end{tabular}


Tabela III Continuação.

\begin{tabular}{|c|c|c|c|c|c|c|}
\hline Espécies & Borda & Araucária & Fase 1 & Fase 2 & Fase 3 & Total \\
\hline Hypolampsis ambiguus (Clark, 1860) & 7 & 0 & 0 & 0 & 0 & 7 \\
\hline Hypolampsis fragilis (Clark, 1860) & 0 & 0 & 0 & 0 & 4 & 4 \\
\hline Hypolampsis nigripes (Clark, 1860) & 1 & 0 & 0 & 0 & 0 & 1 \\
\hline Hypolampsis sp.1 & 0 & 0 & 0 & 1 & 45 & 46 \\
\hline Hypolampsis sp.2 & 0 & 11 & 0 & 0 & 0 & 11 \\
\hline Hypolampsis sp.3 & 7 & 0 & 1 & 0 & 0 & 8 \\
\hline Hypolampsis sp.4 & 0 & 4 & 3 & 0 & 0 & 7 \\
\hline Hypolampsis sp.5 & 0 & 0 & 2 & 0 & 0 & 2 \\
\hline Longitarsus sp.1 & 3 & 3 & 1 & 1 & 4 & 12 \\
\hline Longitarsus sp. 2 & 1 & 0 & 0 & 0 & 0 & 1 \\
\hline Longitarsus sp. 3 & 2 & 0 & 0 & 0 & 0 & 2 \\
\hline Monomacra yena Bechyné, 1957 & 2 & 0 & 22 & 0 & 0 & 24 \\
\hline Monoplatus ocularis Bechyné, 1955 & 45 & 27 & 14 & 48 & 0 & 134 \\
\hline Neodiphaulaca itapiranga (Bechyné, 1957) & 4 & 0 & 0 & 0 & 0 & 4 \\
\hline Neothona prima Bechyné, 1955 & 0 & 2 & 13 & 8 & 0 & 23 \\
\hline Omophoita equestris Fabricius, 1787 & 1 & 0 & 0 & 0 & 0 & 1 \\
\hline Omophoita magniguttis (Bechyné,1955) & 2 & 0 & 0 & 0 & 0 & 2 \\
\hline Omophoita octogutata Fabricius, 1875 & 15 & 5 & 7 & 0 & 0 & 27 \\
\hline Omophoita sexnotata Harold, 1876 & 7 & 0 & 0 & 0 & 0 & 7 \\
\hline Paranaita bilimbata (Baly, 1859) & 2 & 0 & 1 & 0 & 0 & 3 \\
\hline Paranaita crotchi (Blackwelder, 1946) & 0 & 0 & 0 & 0 & 2 & 2 \\
\hline Phyllotrupes violaceomaculatus (Bechyné, 1958) & 3 & 5 & 14 & 138 & 3 & 163 \\
\hline Pyxidaltica variegata (Jacoby, 1880$)$ & 0 & 1 & 0 & 0 & 0 & 1 \\
\hline Rhinotmetus diversipes (Bechyné, 1956) & 3 & 0 & 0 & 0 & 0 & 3 \\
\hline Rhinotmetus sp.1 & 2 & 0 & 1 & 0 & 1 & 4 \\
\hline Rhinotmetus sp.2 & 0 & 1 & 0 & 0 & 0 & 1 \\
\hline Rhinotmetus sp.3 & 0 & 0 & 0 & 1 & 0 & 1 \\
\hline Stegnea inflatipes (Bechyné, 1955) & 0 & 6 & 0 & 23 & 0 & 29 \\
\hline Syphraea multiimpressa Bechyné, 1955 & 0 & 0 & 0 & 2 & 0 & 2 \\
\hline Syphraea olga Bechyné, 1955 & 0 & 1 & 0 & 65 & 0 & 66 \\
\hline Syphraea plaumanni Bechyné, 1955 & 0 & 0 & 0 & 20 & 0 & 20 \\
\hline Syphraea sp. 1 & 0 & 0 & 0 & 44 & 0 & 44 \\
\hline Syphraea sp.2 & 0 & 0 & 0 & 7 & 0 & 7 \\
\hline Syphraea sp.3 & 0 & 0 & 1 & 0 & 0 & 1 \\
\hline Systena tenuis Bechyné, 1954 & 3 & 0 & 0 & 0 & 0 & 3 \\
\hline Systena $\mathrm{sp}$. & 0 & 1 & 0 & 0 & 0 & 1 \\
\hline Thrasygoeus sp. & 1 & 0 & 0 & 0 & 0 & 1 \\
\hline Trichaltica elegantula Baly, 1876 & 3 & 177 & 4 & 6 & 14 & 204 \\
\hline Trichaltica micros Bechyné, 1954 & 1 & 1 & 8 & 3 & 0 & 13 \\
\hline Trichaltica sp. & 1 & 0 & 0 & 0 & 0 & 1 \\
\hline Walterianella argentinensis Jacoby, 1905 & 2 & 0 & 0 & 0 & 0 & 2 \\
\hline Walterianella interruptovittata Jacoby, 1905 & 2 & 0 & 0 & 0 & 0 & 2 \\
\hline Walterianella sp. & 2 & 0 & 0 & 0 & 0 & 2 \\
\hline Wanderbiltiana festiva (Germar, 1824) & 1 & 1 & 0 & 18 & 0 & 20 \\
\hline Wanderbiltiana novoteutoniensis Bechyné, 1955 & 0 & 0 & 0 & 1 & 0 & 1 \\
\hline Wanderbiltiana sejuncta (Harold, 1880) & 1 & 0 & 2 & 0 & 18 & 21 \\
\hline Wanderbiltiana $\mathrm{sp}$. & 0 & 0 & 0 & 2 & 0 & 2 \\
\hline 106 Espécies & 194 & 284 & 404 & 760 & 249 & 1891 \\
\hline
\end{tabular}

estar sofrendo o efeito de massa (Shmida \& Wilson 1985), no qual uma área com grande abundância de determinada espécie, propicia a migração desta para áreas vizinhas. A composição de espécies e a abundância de indivíduos em áreas ecotonais pode ainda ser afetada pela predação e o parasitismo (Yahner 1988).

Índices de diversidade e uniformidade. Através do cálculo dos índices de diversidade de Shannon (H') e Brillouin (HB), os quais geraram valores que ocupam posições equivalentes, a área de Borda foi a que se apresentou mais diversa enquanto que a área de Araucária foi a menos diversa (Tabela IV). As áreas em sucessão apresentaram valores crescentes a medida que o grau de sucessão avança. O índice de Uniformidade de Berger \& Parker (UBP) indicou a área mais conservada, F3, como a mais uniforme e a área de Araucária como a menos. $\mathrm{O}$ alto valor para o índice de Dominância de Berger \& Parker (BP) na área de Araucária deve-se a Trichaltica elegantula Baly, 
Tabela IV. Número de espécies de Alticini (S), abundância (N), índice de diversidade de Shannon (H') e Brillouin (HB), dominância de Berger \& Parker (BP) e uniformidade de Berger \& Parker (UBP) de Alticini, coletados com malaise em cinco áreas no Parque Estadual de Vila Velha, Ponta Grossa, Paraná, de setembro/1999 a agosto/2001.

\begin{tabular}{lcccccc}
\hline Áreas & $\mathrm{S}$ & $\mathrm{N}$ & $\mathrm{H}^{\prime}$ & HB & $\mathrm{BP}$ & UBP \\
\hline Borda & 55 & 194 & 3,29 & 1,29 & 0,23 & 4,35 \\
Araucária & 26 & 284 & 1,67 & 0,67 & 0,62 & 1,61 \\
Fase 1 & 30 & 404 & 2,04 & 0,85 & 0,46 & 2,17 \\
Fase 2 & 40 & 760 & 2,29 & 0,97 & 0,35 & 2,86 \\
Fase 3 & 29 & 249 & 2,67 & 1,09 & 0,18 & 5,55 \\
\hline
\end{tabular}

1876 com 177 exemplares coletados, perfazendo $62,3 \%$ do total coletado nesta área.

Espécies raras e comuns às áreas. Dentre as 106 espécies coletadas, cinco, D. gigia, T. elegantula, P. violaceomaculatus, Monoplatus ocularis Bechyné, 1955 e $S$. olga (Tabela III) foram as mais abundantes perfazendo $54,5 \%$ do total. Setenta e sete $(72,6 \%)$ apresentaram menos de 10 exemplares, 16 $(15,1 \%)$ foram representadas por dois indivíduos (doubletons) e $32(30,2 \%)$ por um único indivíduo (singletons) (Tabela V). A área de Borda apresentou o maior número de singletons (23) e doubletons (12) perfazendo $63,6 \%$ do total coletado nesta área e a área Fase 3, os menores valores, sete e três, respectivamente, representando $34,4 \%$ do total. As áreas, Araucária, Fase 1 e Fase 2, apresentaram uma relativa semelhança entre a soma dos valores percentuais de singletons e doubletons com $49,9 \%, 43,3 \%$ e 47,5\%, respectivamente.

Verifica-se, com isso, que as duas áreas com maior grau de antropização, área de Borda e Araucária, tiveram os maiores números de espécies raras, somando singletons e doubletons. Ganho \& Marinoni (2005) tratando Coleoptera ao nível de espécie, verificaram um resultado mais marcante, onde os percentuais de espécies raras incluindo as únicas (Coddington et al.1996) foram maiores nas áreas mais antropizadas Borda $\mathrm{e}$ Araucária e, nas área em sucessão diminuiram da Fase 1 até a Fase 3.

Morse et al. (1988), ao tratarem da comunidade de Coleoptera que ocupa copas de árvores em Borneo, encontraram 58\% de singletons. Novotný \& Basset (2000), ao estudarem a guilda de insetos herbívoros mastigadores em Nova Guiné, verificaram um percentual de $30 \%$. O valor obtido no presente trabalho foi de $45,3 \%$, intermediário ao dos estudos anteriores que incluem outros grupos além de Alticini. Segundo Novotný \& Basset (2000), muitas vezes um número alto de espécies raras pode não ser real. Segundo esses autores, muitas espécies podem ser "aparentemente" raras devido ao número insuficiente de coletas realizadas, tanto sazonalmente como espacialmente. Isso poderia ocorrer quando as espécies não estivessem em sua melhor época de ocorrência ou deslocadas de seu habitat ou planta hospedeira em que ocorrem preferencialmente.

Verifica-se também que Alticini segue o padrão de outros grupos, onde poucas espécies apresentaram grande número de indivíduos, enquanto que, a maioria é representada por poucos exemplares (Fig. 1). Esse padrão recorrente tem sido comumente encontrado em vários outros trabalhos, nos mais variados grupos dentro de Arthropoda, a partir de diferentes métodos de coleta como fogging (Morse et al. 1988; Santos et al. 2003), rede de varredura (Pinheiro et al. 1998), coleta de serrapilheira (Barbosa et al. 2002) e coleta manual (Coddington et al. 1996).

Somente sete espécies $(6,6 \%)$ foram comuns a todas as áreas - D. gigia, T. elegantula, P. violaceomaculatus, Heikertingerella ferruginea Duvivier, 1889, Acanthonycha costatipennis Jacoby, 1905, Acanthonycha chloroptera (Germar, 1824) e Longitarsus sp.1, enquanto que 69 espécies $(65,1 \%)$ foram exclusivas, sendo coletadas somente em uma das cinco áreas. Apesar destas sete espécies terem ocorrido em todas as áreas, pode-se dizer que as mesmas são características das áreas onde ocorreram em maior abundância (Tabela III). A exemplo, D. giga é característica das áreas Fase 1 e Fase 2, enquanto sua ocorrência no restante das áreas pode ser considerada esporádica.

Curva de acumulação de espécies. A curva com os valores do número acumulado de espécies capturadas mensalmente ao longo dos dois anos para Vila Velha (Fig. 2) mostra que, no primeiro ano $79 \%$ do total de espécies já haviam sido coletadas, sendo que nos cinco primeiros meses já se havia coletado mais da metade das espécies, $64 \%$. Em março a curva tendeu a uma assíntota, porém em outubro do segundo ano houve um incremento com a captura de novas espécies que seguiu até janeiro, a partir de onde a curva tende a se estabilizar. $\mathrm{O}$ incremento a partir de outubro coincide com o segundo ciclo sazonal (primavera), característico de espécies de clima temperado. Quando analisado somento o primeiro ano, nos três primeiros meses já haviam sido capturadas $60,7 \%$ das espécies. Este percentual foi próximo aos obtidos por Marinoni \& Dutra (1996), Marinoni et al. (1997, 1999), de 66 a 71\%, nos levantamentos ao nível de família de grupos de lepidópteros que são essencialmente herbívoros como os Alticini, opondose ao percentual de 44 a $57 \%$ encontrado para a análise de Coleoptera ao nível de espécie por Ganho \& Marinoni (2005).

Para cada uma das áreas foi possível verificar que o padrão

Tabela V. Número total de espécies de Alticini, singletons e doubletons, e seus percentuais, capturadas com malaise, em cinco áreas no Parque Estadual de Vila Velha, Ponta Grossa, Paraná, de setembro/1999 a agosto/2001.

\begin{tabular}{lccccrr}
\hline Espécies & Borda & Araucária & Fase 1 & Fase 2 & Fase 3 & Total \\
\hline Total & 55 & 26 & 30 & 40 & 29 & 106 \\
Singleton & $23(41,8 \%)$ & $8(30,7 \%)$ & $10(33,3 \%)$ & $15(37,5 \%)$ & $7(24,1 \%)$ & $32(30,2 \%)$ \\
Doubleton & $12(21,8 \%)$ & $5(19,2 \%)$ & $3(10,0 \%)$ & $4(10,0 \%)$ & $3(10,3 \%)$ & $16(15,1 \%)$ \\
\hline
\end{tabular}


Tabela VI. Valores observados (So) e estimados da riqueza de espécies de Alticini, gerados através dos estimadores Chao 1, Chao 2, Jack-knife1, Jack-knife2, Bootstrap e Michaelis-Menten, para cada uma das áreas e para Vila Velha, a partir de dados semanais, coletados com malaise no Parque Estadual de Vila Velha, Ponta Grossa, Paraná, de setembro/1999 a agosto/2001.

\begin{tabular}{|c|c|c|c|c|c|c|c|c|}
\hline & Áreas & So & Chao 1 & Chao 2 & Jack-knife1 & Jack-knife2 & Bootstrap & $\begin{array}{r}\text { Michaelis- } \\
\text { Menten } \\
\end{array}$ \\
\hline & Borda & 39 & $58,32 \pm 12,40$ & $58,32 \pm 12,40$ & $60,57 \pm 4,92$ & $71,36 \pm 2,50$ & $48,58 \pm 0,94$ & 75,68 \\
\hline 9 & Araucária & 16 & $23,25 \pm 10,27$ & $28,50 \pm 20,18$ & $24,82 \pm 3,04$ & $31,59 \pm 1,40$ & $19,60 \pm 0,64$ & 21,05 \\
\hline 9 & Fase 1 & 28 & $35,25 \pm 10,27$ & $40,50 \pm 20,18$ & $36,82 \pm 2,70$ & $43,59 \pm 1,42$ & $31,86 \pm 0,58$ & 31,71 \\
\hline- & Fase 2 & 30 & $77,25 \pm 111,56$ & $61,11 \pm 43,99$ & $43,73 \pm 3,73$ & $55,30 \pm 1,77$ & $35,49 \pm 0,62$ & 33,66 \\
\hline 0 & Fase 3 & 23 & $30,25 \pm 10,27$ & $32,28 \pm 12,45$ & $31,82 \pm 2,70$ & $37,65 \pm 1,37$ & $26,84 \pm 0,53$ & 29,91 \\
\hline \multirow[t]{2}{*}{0} & Vila Velha & 84 & $119,27 \pm 18,17$ & $126,59 \pm 21,72$ & $118,33 \pm 6,06$ & $139,73 \pm 3,78$ & $98,82 \pm 1,22$ & 94,79 \\
\hline & Borda & 36 & $64,76 \pm 23,89$ & $60,62 \pm 19,27$ & $54,63 \pm 5,05$ & $67,24 \pm 2,72$ & $43,95 \pm 0,94$ & 61,28 \\
\hline 0 & Araucária & 19 & $22,93 \pm 6,48$ & $22,12 \pm 4,80$ & $24,88 \pm 3,33$ & $26,88 \pm 0,98$ & $31,96 \pm 0,32$ & 28,76 \\
\hline 0 & Fase 1 & 10 & $12,22 \pm 5,29$ & $13,61 \pm 7,55$ & $14,90 \pm 2,52$ & $17,82 \pm 0,99$ & $12,08 \pm 0,51$ & 11,58 \\
\hline- & Fase 2 & 28 & $39,56 \pm 14,84$ & $39,56 \pm 14,84$ & $37,80 \pm 3,71$ & $44,59 \pm 1,51$ & $32,09 \pm 0,66$ & 29,89 \\
\hline 0 & Fase 3 & 22 & $31,28 \pm 12,45$ & $31,28 \pm 12,45$ & $30,82 \pm 3,04$ & $36,65 \pm 1,32$ & $25,78 \pm 0,38$ & 26,95 \\
\hline \multirow[t]{2}{*}{$\underline{1}$} & Vila Velha & 76 & $108,02 \pm 19,13$ & $103,08 \pm 15,62$ & $102,48 \pm 6,34$ & $117,13 \pm 2,84$ & $87,75 \pm 1,32$ & 87,96 \\
\hline & Borda & 55 & $74,53 \pm 12,13$ & $74,53 \pm 12,13$ & $77,78 \pm 5,26$ & $88,68 \pm 1,54$ & $65,53 \pm 0,52$ & 83,10 \\
\hline 9 & Araucária & 26 & $30,78 \pm 5,92$ & $30,08 \pm 4,93$ & $33,92 \pm 3,05$ & $35,94 \pm 1,00$ & $29,99 \pm 0,35$ & 34,05 \\
\hline 9 & Fase 1 & 30 & $41,56 \pm 14,84$ & $48,94 \pm 28,64$ & $40,89 \pm 3,12$ & $49,74 \pm 0,98$ & $34,61 \pm 0,31$ & 32,81 \\
\hline- & Fase 2 & 40 & $60,30 \pm 20,90$ & $60,30 \pm 20,90$ & $53,86 \pm 3,83$ & $64,68 \pm 1,08$ & $45,14 \pm 0,41$ & 39,77 \\
\hline 0 & Fase 3 & 29 & $34,47 \pm 8,28$ & $36,25 \pm 10,27$ & $36,92 \pm 2,70$ & $41,86 \pm 1,13$ & $32,64 \pm 0,39$ & 33,36 \\
\hline 1 & Vila Velha & 106 & $135,23 \pm 14,97$ & $139,06 \pm 16,85$ & $138,68 \pm 7,07$ & $156,48 \pm 2,73$ & $120,82 \pm 1,14$ & 113,31 \\
\hline
\end{tabular}

geral encontrado para Alticini em Vila Velha foi em função principalmente das espécies capturadas na área de Borda na qual durante os cinco primeiros meses de coleta houve um acentuado acréscimo na captura de novas espécies (Fig. 2). Nesse período foram coletadas cerca de $62 \%$ do total de espécies. Em outubro/2000 houve um novo acréscimo que continuou até janeiro, onde a curva praticamente se estabilizou. A área de Araucária foi a que apresentou maior incremento na captura de novas espécies durante o segundo ano, 39\%. Para a área Fase 1, os três primeiros meses foram suficientes para coletar $76 \%$ das espécies e em fevereiro/2000 a curva já havia praticamente se estabilizado, com $90 \%$ das espécies capturadas. As áreas mais conservadas, Fase 2 e Fase 3, tiveram no primeiro ano $75 \%$ e $79 \%$ de suas espécies coletadas, respectivamente, sendo que nos cinco primeiros meses foram coletadas $50 \%$ das espécies da área Fase 2 e $62 \%$ das espécies da Fase 3. Na área Fase 2 ocorreu um pequeno incremento de espécies em julho/2000 que permaneceu até dezembro/2000.

Estimativa de riqueza. Na definição de estratégias para a priorização de áreas para conservação, a riqueza de espécies é a forma mais simples e fácil de se medir a diversidade de um local (Palmer 1990; Moreno \& Halffter 2000; Halffter et al. 2001; Chao 2004). Porém, em muitos casos, é impraticável enumerar diretamente as espécies (Colwell 2004). Daí a importância dos estimadores de riqueza como mais um elemento para a caracterização das áreas, pois, por melhor que seja o método de coleta empregado, dificilmente todas as espécies serão capturadas.

Dentre os métodos utilizados para calcular a estimativa de riqueza de espécies, Jack-knife 2 foi o que resultou nos maiores valores estimados para todas as áreas quando analisado todo o período de coletas, enquanto que Bootstrap resultou nos menores valores, com exceção das áreas Fase 1 e Fase 2, que tiveram seus menores valores estimados por Michaelis-Menten (Tabela VI). Na área de Borda, onde foram capturadas 55 espécies de Alticini, a riqueza estimada por Jack-knife 2 foi de 88,68 espécies, ou seja, foram coletados $62 \%$ do total de espécies estimadas, enquanto por Bootstrap foi de 65,53 espécies, com $83,9 \%$ coletados. Para as outras áreas os maiores valores gerados por Jack-knife 2 e os menores gerados por Bootstrap e Michaelis-Menten foram, respectivamente: área de Araucária, 35,94 e 29,99 espécies, com 26 espécies efetivamente capturadas; Fase 1, 49,74 e 32,81 espécies, para 30 espécies capturadas; Fase 2, 64,68 e 39,77 espécies, para 40 espécies capturadas e Fase 3, 41,86 e 32,64 espécies para 29 espécies capturadas (Tabela VI). Portanto as espécies capturadas representaram entre $60 \%$ e $72 \%$ dos maiores valores estimados e entre $84 \%$ e $89 \%$ dos menores valores estimados.

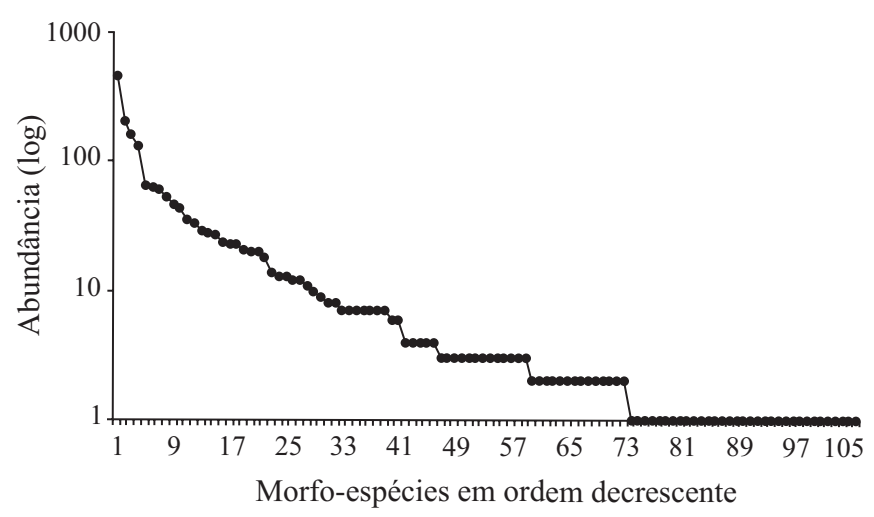

Fig. 1. Abundância de Alticini em ordem decrescente das morfo-espécies, em escala logarítmica, coletados com malaise, no Parque Estadual de Vila Velha, Ponta Grossa, Paraná, de setembro/1999 a agosto/2001. 


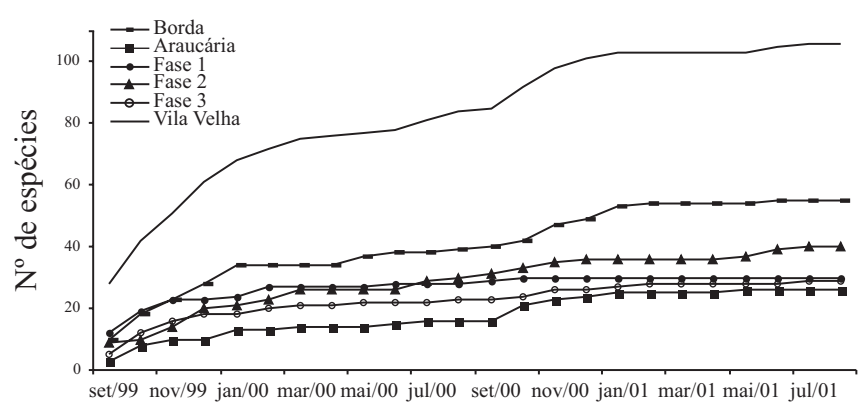

Fig. 2. Número acumulado de espécies de Alticini, para Vila Velha e para cada uma das áreas, capturadas mensalmente com malaise no Parque Estadual de Vila Velha, Ponta Grossa, Paraná, de setembro/1999 a agosto/ 2001 .

Com base exclusivamente nos dados do primeiro ano de coleta (52 amostras), de todas as áreas em conjunto, verificase que Bootstrap e Michaelis-Menten estimaram menos do que realmente foi coletado com a continuidade das coletas (104 amostras), 98,82 e 94,79, respectivamente, enquanto que foram realmente coletadas 106 espécies (Tabela VI). Este mesmo comportamento foi observado para a estimativa da Fase 2, por Michaelis-Menten, (104 amostras), onde o valor estimado foi de 39,77 e o número de espécies coletadas, 40. Segundo Palmer (1990), quando um estimador gera um valor menor que o observado, o mesmo pode ser considerado um estimador ruim.

Considerando os diferentes estimadores e o número de espéceis já capturadas, podem ser esperadas de sete (Michaelis-Menten) a 50 (Jack-knife 2) novas espécies para Vila Velha.

As estimativas, de modo geral, refletiram a riqueza realmente observada nas áreas. Considerando a maioria dos estimadores, a área de Borda apresentou as maiores estimativas de riqueza durante os dois anos em conjunto e separadamente. A menor estimativa foi para a área de Araucária no primeiro ano e no conjunto dos anos, e para a área Fase 1 no segundo ano (Tabela VI). Ao contrário, Ganho \& Marinoni (2005) verificaram que a

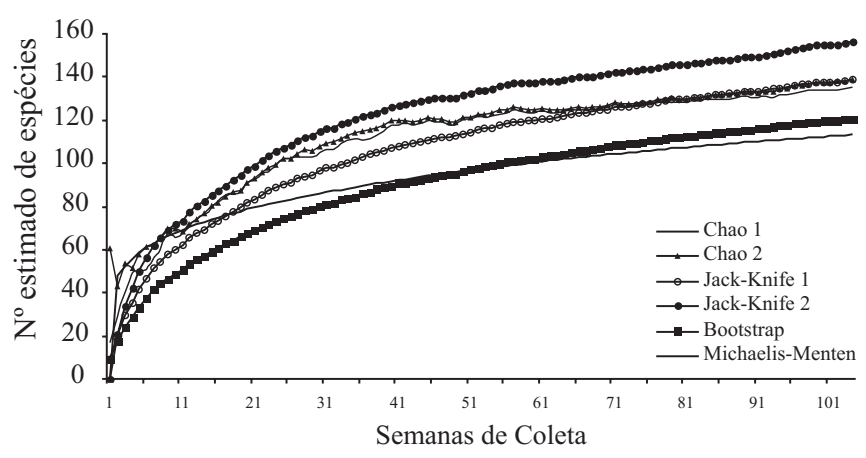

Fig. 3. Número estimado de espécies de Alticini utilizando os métodos Chao 1, Chao 2, Jack-knife 1, Jack-knife 2, Bootstrap e MichaelisMenten, para Vila Velha, gerados a partir de dados semanais, coletados com malaise, no Parque Estadual de Vila Velha, Ponta Grossa, Paraná, de setembro/1999 a agosto/2001. área Fase 1 foi a que teve o maior valor estimado e a Fase 3, a mais conservada, o menor valor. Indicaram ainda uma tendência, não observada no presente trabalho, dos valores estimados proporcionalmente declinarem das áreas menos conservadas para as mais conservadas.

O gráfico da estimativa de riqueza de espécies para Vila Velha mostra que as curvas geradas pelos estimadores tendem a uma assíntota (Fig. 3). Segundo Colwell \& Coddington (1994), se o estimador atingir um platô estável, ainda que a curva seja ascendente devido às últimas coletas, o levantamento pode ter sido adequado e, com o aumento de coletas geralmente os estimadores tornam-se mais precisos (Colwell 2004).

Dessa forma, para Vila Velha de um modo geral, o levantamento foi adequado e o número de coletas (104) parece suficiente para termos uma estimativa segura do número de espécies que ali ocorrem.

\section{CONCLUSÕES}

A área Fase 2, em estágio intermediário a avançado de sucessão, apresentou a maior abundância de Coleoptera, Chrysomelidae e Alticini. A área de Borda foi a mais rica em espécies, provavelmente por se tratar de um ecótono e também devido a alta produtividade do local e, a área de Araucária, provavelmente por ser mais homogênea, apresentou a menor riqueza.

As áreas mais conservadas, em estágio mais avançado de sucessão, apresentaram menor proporção de Chrysomelidae, indicando que este grupo possui alto potencial indicador de qualidade ambiental, porém Alticini não apresentou uma diminuição gradativa em sua proporção de áreas menos conservadas para as mais conservadas, mas apresentou sua menor proporção na área mais conservada.

O estimador Jack-knife 2, que gerou os maiores valores, indicou a possibilidade de serem coletadas mais 50 espécies em Vila Velha com a continuidade dos estudos.

Os estimadores Bootstrap e Michaelis-Menten mostraramse menos seguros para a estimativa de espécies de Alticini, sendo necessário que sejam melhor avaliados quanto a sua validade.

Agradecimentos. A Luciano de Azevedo Moura pelo auxílio na identificação do material e a Fernando Mayer por permitir o acesso à coleção do Museu Anchieta do Rio Grande do Sul. Ao CNPq pela concessão das Bolsas.

\section{REFERÊNCIAS}

Barbosa, M. G. V.; C. R. V. Fonseca; P. M. Hammond \& N. E. Stork. 2002. Diversidade e similaridade entre habitats com base na fauna de Coleoptera de serrapilheira de uma floresta de terra firme da Amazônia Central, pp. 69-83. In: Costa, C.; S. A. Vanin; J. M. Lobo \& A. Melic (Eds). Proyecto de Red Iberoamericana de Biogeografia y Entomología Sistemática - PrIBES. Vol 2, Sociedad Entomológica Aragonesa, Zaragosa, España. 327p.

Chao, A. 2004. Species richness estimation. In: Balakrishnan, N.; C. B. Read \& B. Vidakovic (Eds). Encyclopedia of Statistical Sciences. Wiley, New York.

Coddington, J. A.; L. H. Young \& F. A. Coyle. 1996. Estimating spider 
species richness in a southern appalachian cove hardwood forest. The Jounal of Arachnology 24: 111-128.

Colwell, R. K. 2004. EstimateS: Statistical Estimation of Species Richness and Shared Species from Samples, Version 7.00. User's guide and application published at: http://viceroy.eeb.uconn.edu/ estimates. Acesso em: 09 nov. 2004.

Colwell, R. K. \& J. A. Coddington. 1994. Estimating terrestrial biodiversity through extrapolation. Philosophical Transactions of Royal Society of London (Ser. B) 345: 101-118.

Didham, R. K.; J. H. Lawton; P. M. Hammond \& P. Eggleton. 1998a. Trophic structure stability and extinction dynamics of beetles (Coleoptera) in tropical forest fragments. Philosophical Transactions of Royal Society of London 353: 437-451.

Didham, R. K.; P. M. Hammond; J. H. Lawton; P. Eggleton \& N. E. Stork. 1998b. Beetles species responses to tropical forest fragmentation. Ecological Monographs 68: 295-323.

Farrell, B. D. \& T. L. Erwin. 1988. Leaf-beetle community structure in an amazonian rainforest canopy, pp. 73-90. In: Jolivet, P.; E. Petitpierre \& T. Hsiao (Eds). Biology of Chrysomelidae. Kluwer Academic Publ., Dordrecht. 608p.

Furth, D. G. 1988. The jumping apparatus of flea beetles (Alticinae), pp. 285-297. In: Jolivet, P.; E. Petitpierre \& T. Hsiao (Eds). Biology of Chrysomelidae. Kluwer Academic Publ., Dordrecht. $608 \mathrm{p}$.

Ganho, N. G. \& R. C. Marinoni. 2003. Fauna de Coleoptera no Parque Estadual de Vila Velha, Ponta Grossa, Paraná, Brasil. Abundância e riqueza das famílias capturadas através de armadilhas malaise. Revista Brasileira de Zoologia 20: 727-736.

Ganho, N. G. \& R. C. Marinoni. 2005. A diversidade inventarial de Coleoptera (Insecta) em uma paisagem antropizada do Bioma Araucária. Revista Brasileira de Entomologia 49: 535-543.

Halffter, G.; C. E. Moreno \& E. O. Pineda. 2001. Manual para evaluación de la biodiversidad en Reservas de la Biosfera. M\&T - Manuales y Tesis SEA, vol. 2. Zaragoza, 80p.

Hutcheson, J. 1990. Characterization of terrestrial insect communities using quantified, Malaise-trapped Coleoptera. Ecological Entomology 15: 143-151.

Magurran, A. E. 2004. Measuring Biological Diversity. Blackwell Science Ltd, Oxford, 256p.

Marinoni, R. C. \& R. R. C. Dutra. 1996. Levantamento da fauna entomológica no Estado do Paraná. II. Ctenuchidae (Lepidoptera). Revista Brasileira de Zoologia 13: 435-461.

Marinoni, R. C. \& R. R. C. Dutra. 1997. Famílias de Coleoptera capturadas com armadilha Malaise em oito localidades do Estado do Paraná, Brasil. Diversidades alfa e beta. Revista Brasileira de Zoologia 14: 751-770.

Marinoni, R. C.; R. R. C. Dutra \& M. M. Casagrande. 1997. Levantamento da fauna entomológica no Estado do Paraná. III. Saturniidae (Lepidoptera). Revista Brasileira de Zoologia 14: 473-495.

Marinoni, R. C.; R. R. C. Dutra \& O. H. H. Mielke. 1999. Levantamento da fauna entomológica no Estado do Paraná. IV. Sphingidae (Lepidoptera). Diversidade alfa e estrutura de comunidade. Revista Brasileira de Zoologia 16: 223-240.

Marinoni, R. C. \& N. G. Ganho. 2003. A fauna de Coleoptera em áreas com diferentes condições florísticas no Parque Estadual de Vila Velha, Ponta Grossa, Paraná, Brasil. Abundância e riqueza das famílias capturadas através de armadilhas de solo. Revista Brasileira de Zoologia 20: 737-744.

Moreno, C. E. \& G. Halffter. 2000. Spatial and temporal analysis of a, $\mathrm{b}$ and $\mathrm{g}$ diversities of bats in a fragmented landscape. Biodiversity and Conservation 10: $367-382$.

Morris, M. G. 1980. Insects and the Environment in the United Kingdom. Atti XII Congresso Nazionale Italiano di Entomologia, Roma. 203-235p.

Morse, D. R.; N. E. Stork \& J. H. Lawton. 1988. Species number, species abundance and body length relationships of arboreal beetles in Bornean lowland rain forest trees. Ecological Entomology 13: $25-37$.

Novotný, V. \& Y. Basset. 2000. Rare species in communities of tropical insect herbivores: pondering the mystery of singletons. Oikos 89: $564-572$.

Novotný, V.; Y. Basset; G. A. Samuelson \& S. E. Miller. 1999. Host use by chrysomelid beetles feeding on Moraceae and Euphorbiaceae in New Guinea, pp. 545-555. In: Cox, M. L. Advances in Chrysomelidae Biology 1. Backhuys Publishers, Leiden, The Netherlands. 671p.

Oliveira, E. B.; R. D. Miyazaki \& W. Severi. 1998. Cálculo de fatorial e seu uso no índice de Brillouin. Revista Brasileira de Biologia 58: $37-341$.

Palmer, M. W. 1990. The estimation of species richness by extrapolation. Ecology 71: 1195-1198.

Pinheiro, F.; I. R. Diniz \& K. Kitayama. 1998. Comunidade local de Coleoptera em Cerrado: Diversidade de espécies e tamanho do corpo. Anais da Sociedade Entomológica do Brasil 27: 543550 .

Santos, G. B. dos; M. I. Marques; J. Adis \& C. R. DeMusis. 2003. Artrópodos associados à copa de Attalea phalerata Mart. (Arecaceae), na região do Pantanal de Poconé, Mato Grosso, Brasil. Revista Brasileira de Entomologia 47: 211-224.

Scherer, G. 1988. The origins of the Alticinae, pp. 115-130. In: Jolivet, P.; E. Petitpierre \& T. Hsiao (Eds). Biology of Chrysomelidae. Kluwer Academic Publ., Dordrecht. 608p.

Shmida, A. \& M. R. Wilson.1985. Biological determinants of species diversity. Journal of Biogeography 12: 1-20.

Takizawa, H. 1994. Seasonal changes in leaf beetle fauna of a warm temperate lowland in Japan, pp. 511-525. In: Jolivet, P. H.; M. L. Cox \& E. Petitpierre (Eds). Novel aspects of the biology of Chrysomelidae. Kluwer Academic Publishers, Netherlands. 582p.

Townes, H. 1972. A light-weight malaise trap. Entomological News 83: $239-247$.

Wagner, T. 1999. Arboreal chrysomelid community structure and faunal overlap between different types of forests in Central África, pp. 247-270. In: Cox, M. L. (Ed.). Advances in Chrysomelidae Biology 1. Backhuys Publishers, Leiden, The Netherlands. 671p.

Wolda, H. 1978. Seasonal fluctuations in rainfall, food and abundance of tropical insects. Journal of Animal Ecology 47: 369-381.

Yahner, R. H. 1988. Changes in wildlife communities near edges. Conservation Biology 2: 333-339. 